\title{
Expressions of ABCG2, CD133, and Podoplanin in Salivary Adenoid Cystic Carcinoma
}

\author{
Wuwei Li, ${ }^{1}$ Ryo Tamamura, ${ }^{2}$ Bo Wang, ${ }^{3}$ Qigui Liu, ${ }^{4}$ Han Liu, ${ }^{1}$ Tingjiao Liu, \\ Naoki Katase, ${ }^{5}$ Jing Xiao, ${ }^{1}$ and Hitoshi Nagatsuka ${ }^{5}$ \\ ${ }^{1}$ Medical College of Stomatology, Dalian Medical University, 9 Western Section, Lvshun South Street, Dalian 116044, China \\ ${ }^{2}$ Department of Histology, Nihon University School of Dentistry at Matsudo, 2-870-1 Sakaemachi-nishi, Matsudo 271-8587, Japan \\ ${ }^{3}$ Comprehensive Transplant Center, Department of Surgery, Feinberg School of Medicine, Northwestern University, \\ 300 E. Superior Street, Chicago, IL 60611, USA \\ ${ }^{4}$ Department of Health Statistics, Dalian Medical University, 9 Western Section, Lvshun South Street, Dalian 116044, China \\ ${ }^{5}$ Department of Oral Pathology and Medicine, Graduate School of Medicine, Dentistry and Pharmaceutical Sciences, \\ Okayama University, 2-5-1 Shikata-cho, Okayama 700-8525, Japan
}

Correspondence should be addressed to Hitoshi Nagatsuka; jin@md.okayama-u.ac.jp

Received 11 December 2013; Accepted 13 March 2014; Published 6 April 2014

Academic Editor: Jun Liao

Copyright (C) 2014 Wuwei Li et al. This is an open access article distributed under the Creative Commons Attribution License, which permits unrestricted use, distribution, and reproduction in any medium, provided the original work is properly cited.

\begin{abstract}
Adenoid cystic carcinoma (ACC) is one of the most common salivary gland malignant tumors with a high risk of recurrence and metastasis. Current studies on cancer stem cells (CSCs) have verified that CSCs are the driving force behind tumor initiation and progression, suggesting that new cancer therapies may be established by effectively targeting and killing the CSCs. The primary goal of this study is to investigate the expression patterns of ABCG2, CD133, and podoplanin in ACC of minor salivary glands by immunohistochemistry analysis. We found that ABCG2 was weakly expressed in normal looking salivary gland tissues. A significant upregulation of ABCG2 expression in ACC was observed with a similar expression pattern of Ki-67. CD133 was detected in apical membrane of epithelial cells and podoplanin was expressed positively in myoepithelial cells of both normal looking tissue and ACC. However, no significant difference was found of the expression pattern of CD133 and podoplanin between normal looking tissues and ACC. Our observations suggest that CSCs may exist in quiescent cells with ABCG2 positive staining, which are surrounded by cells with positive expression of ABCG2 and Ki-67 in ACC, and costaining with ABCG2 and Ki-67 may help predict the location of CSCs.
\end{abstract}

\section{Introduction}

Adenoid cystic carcinoma (ACC) is one of the most common malignant neoplasms that affects either the major or minor salivary glands of the oral cavity with high risk of recurrence and metastasis [1]. About $50 \%$ of ACCs occur in the hard palate and rarely take place in other intraoral sites including the lower lip, retromolar/tonsillar pillar region, sublingual gland, and buccal mucosa [2]. Currently, surgery and radiotherapy are the most effective treatments, but the outcomes remain unsatisfying [3]. In many cases, ACC shows an indolent clinical course with a considerable risk of local relapse and late distant metastases to lung or bone, which occasionally causes fatal outcome after the first episode [3].
The hypothesis of cancer stem cells states that the tumor formation and growth likely depend on a small subset of tumor cells, known as cancer stem cells (CSCs) or tumorinitiating cells (TICs), which might be the origin of neoplasia [4]. CSCs have been isolated from some types of solid tumors, such as breast, brain, prostate, melanoma, colon, pancreas, head/neck, liver, and gastric cancers [5-8], and they are recognized to be more resistant to radiotherapy and chemotherapy and additionally invasive than normal tumor cells. If CSCs can be targeted by specific CSC markers and killed efficiently, the outcomes of current clinical cancer treatments may be improved [9]. Several putative stem cell markers, such as CD44, CD24, CD166, EpCAM, ABCG2, CD133, and podoplanin, are commonly studied in identifying 
and isolating the CSCs from the solid tumors; however, subsequent studies demonstrated that the choice of CSC markers differs among cancer originations [10-15].

ATP-binding cassette, subfamily G, member 2 (ABCG2) is a member of the superfamily of ATP-binding cassette transporter proteins. Immunohistochemical analysis showed that ABCG2 was expressed ubiquitously in normal and tumor tissues [16]. Zhou et al. [17] found that the expression of $A B C G 2$ gene is an important determinant and a molecular marker for side population-enriched stem cells (SP cells), and this feature was considered to be related to CSCs [18]. Kim et al. also reported that ABCG2 is highly expressed in SP cells and its expression is strongly correlated with SP phenotype [10]. ABCG2 can be expressed in stem cells isolated from both normal and tumor tissues, which further indicates its essential role in stem cell biology [19].

CD133 (prominin-1), a cell-surface glycoprotein carrying five transmembrane domains, was firstly identified as a marker for a subpopulation of CD34-positive haematopoietic stem cells which are derived from human fetal liver, fetal bone marrow, and peripheral blood [20]. CD133 was initially regarded as a specific marker of hematopoietic stem cell and later found in neural stem cells, epidermal stem cells, and endothelial progenitor cells (EPCs) [17, 21, 22]. Previous studies have identified and isolated a putative CSC population from the brain [23], prostate [24], liver [25], lung [26], and colon [27] cancers using CD133 antibodies. In oral CSClike cells isolated from primary tumor cells and cell lines, scientists found an improved migration and tumorigenicity behavior of these cells with a higher CD133 expression level [28]. CD133-positive cells also showed an enhanced clonogenicity, invasiveness, and tumorigenicity compared to those CD133-negative cells [29].

Podoplanin is a mucin-like transmembrane glycoprotein that is highly and specifically expressed in lymphatic endothelial cells of normal tissues and tumors [30]. Recent studies have reported that podoplanin-positive cells in tumors act as mediators of metastasis and invasion [31-33]. Podoplanin is also reported as a potential CSC marker for squamouscell carcinoma (SCC). Atsumi et al. found that A431 cells (a SCC cell line) consisted of both podoplanin-positive and podoplanin-negative cells, in which podoplanin-negative cells only produced podoplanin-negative cells but podoplanin-positive cells generated both podoplanin-positive and -negative cells, and podoplanin-positive cells showed higher tumorigenicity [34]. Rahadiani et al. reported that the esophageal squamous-cell carcinoma (ESCC) showed defective invasion and tumorigenic activities after the podoplanin expression were knocked down in esophageal [35].

Relative studies in identifying CSCs markers in ACC are still in the early stage. In this study, we examined the expression patterns of ABCG2, CD133, and podoplanin in ACC of minor salivary glands by immunohistochemical analysis. Meanwhile, Ki-67, a nuclear protein associated with cellular proliferation, was used to evaluate the proliferation of the tumor cells.

\section{Materials and Methods}

2.1. Tissue Samples. Archival paraffin-embedded tissue blocks of 25 ACC cases (9 men, 16 women; mean age: 60.72 years; range: 32-78 years) were randomly selected. Ten of these samples included normal looking tissues. These samples were diagnosed at Okayama University Hospital Surgical Pathology Unit (Okayama, Japan) from 1980 to 2005. All specimens were fixed with $10 \%$ neutral buffered formalin and embedded in paraffin. Histological diagnosis was carried out by routine hematoxylin and eosin ( $\mathrm{H}$ and $\mathrm{E})$ staining, according to World Health Organization (WHO) histological typing of ACC. Clinical and histopathological characteristics of the patients are shown in Table 1. Ten normal looking salivary gland tissues were used as the control group in this study. Informed consents were obtained from the patients and the experiment was approved by the Research Ethics Board of Okayama University.

2.2. Immunohistochemistry Staining. Antibodies against ABCG2 (BXP-21) (1:50; mouse monoclonal; Abcam, Cambridge, UK), CD133 (1:300; rabbit polyclonal; Abcam), podoplanin (1:50; mouse monoclonal; DAKO, Carpinteria, CA) and Ki-67 (1:50; mouse monoclonal; DAKO) were used in this study.

For immunohistochemical staining, serial sections of $4 \mu \mathrm{m}$ thickness were deparaffinized in xylene and rehydrated in decreasing concentrations of ethanol. Endogenous peroxidase activity was blocked by immersing the sections in $3 \% \mathrm{H}_{2} \mathrm{O}_{2}$ with methanol for $30 \mathrm{~min}$. For antigen retrieval, sections were boiled in $10 \mathrm{mmol} / \mathrm{L}$ citrate buffer $(\mathrm{pH} 6.0$ ) for $15 \mathrm{~min}$ in a pressure cooker. After treatment with protein block serum at room temperature, sections were covered with primary antibodies and incubated at $4^{\circ} \mathrm{C}$ overnight. For secondary antibody, DAKO Cytomation Envision+SystemHRP kit (AEC) was used according to the manufacturer's instructions. Antibody reactions were stained with $3,3^{\prime}$ diaminobenzidine and counterstained with hematoxylin. For the negative control, sections were incubated with PBS instead of the primary antibodies.

2.3. Assessment Standard. ABCG2, CD133, and podoplanin were detected in the cytoplasm and/or cell membrane. Ten areas were selected randomly in each section under high power magnification $(\times 400)$. Also, 100 cells were selected randomly in each area so that a total of 1000 cells were analyzed for counting the positive cells. Positive staining intensity for cancer stem cell markers was classified as negative: $-(<30 \%)$, weak: $+(30-49 \%)$, moderate: $++(50-74 \%)$, and strong staining: $+++(\geq 75 \%)$. For Ki-67 staining, the nucleus was stained with brown color and positive staining intensity was classified as negative: $-(<5 \%)$, weak: $+(5-9 \%)$, moderate: $++(10-14 \%)$, and strong staining: $+++(\geq 15 \%)$. No false positive or negative staining was encountered in the positive or negative controls, respectively.

2.4. Statistical Analysis. Statistical analyses were performed with SPSS version 18.0 for Windows. Wilcoxon test was used 
TABLE 1: Clinical and histopathological data of 25 patients with ACC.

\begin{tabular}{lcccc}
\hline Number & Gender & Age (years) & Primary site & Histological pattern \\
\hline 1 & F & 78 & Mouth floor & Cribriform \\
2 & F & 62 & Mouth floor & Cribriform \\
3 & F & 42 & Mouth floor & Cribriform \\
4 & F & 70 & Mouth floor & Cribriform \\
5 & F & 78 & Mouth floor & Cribriform \\
6 & F & 65 & Mouth floor & Cribriform \\
7 & F & 58 & Mouth floor & Cribriform with tubular \\
8 & F & Mouth floor & Cribriform with tubular \\
9 & M & 66 & Mouth floor & Cribriform with solid \\
10 & F & Mouth floor & Cribriform with solid \\
11 & M & Mouth floor & Cribriform with solid \\
12 & M & 57 & Palate & Cribriform \\
13 & M & 75 & Palate & Cribriform \\
14 & M & 77 & Palate & Cribriform \\
15 & F & Palate & Cribriform \\
16 & F & 62 & Palate & Cribriform \\
17 & F & Palate & Cribriform \\
18 & F & 66 & Palate & Cribriform \\
19 & F & Palate & Cribriform \\
20 & M & 34 & Palate & Cribriform with tubular \\
21 & M & 71 & Palate & Cribriform with tubular \\
22 & F & 32 & Palate & Cribriform with tubular \\
23 & M & 54 & Palate & Cribriform with solid \\
24 & M & F & Buccal mucosa & Cribriform \\
25 & & Buccal mucosa & Cribriform \\
\hline
\end{tabular}

to determine the intensity of marker expression and Fisher's exact test was used to determine the positive percentage of tumor cells and clinicopathological characteristics. Correlations between different markers and Ki-67 expression intensities in different locations of ACC and normal looking tissues were assessed by Spearman's rank correlation analysis. All tests were two-tailed and the differences were considered statistically significant when $P<0.05$.

\section{Results}

3.1. Expression of ABCG2, CD133, Podoplanin, and Ki-67 in Normal Looking Tissues. Immunopositivity for ABCG2 was observed in the cytoplasm and cell membrane of acinar epithelium cells, ductal cells, and myoepithelial cells in 6 cases and the staining intensities were weak in all 6 samples (Figure 1(a); Table 2). A small number of ABCG2-positive cells were present in the capillary endothelium. CD133positive expressions were localized only in apical membrane of acinar epithelial cells and ductal cells in all 10 samples (Figure 1(b)) in which 5 cases showed moderate staining intensity and 5 cases showed strong intensity. Podoplaninpositive expressions were found in the cytoplasm and cell membrane of myoepithelial cells in just 1 case with weak staining intensity (Figure 1(c)). Expression of Ki-67 was negative in all 10 cases (Figure 1(d)).

\subsection{Expression of ABCG2, CD133, Podoplanin, and $\mathrm{Ki}-67$ in $\mathrm{ACC}$}

ABCG2. ABCG2-positive stainings could be detected in 23 cases including 1 case of weak staining intensity, 7 cases of moderate intensity, and 15 cases of strong intensity, and the positive expression percentage was $92 \%(P=0.043)$ (Table 2). The tumor cells showed strong ABCG2 staining in the invasive front of cancer nests and the intensity in peripheral zone was stronger than the center zone. The intensity of ABCG2 staining was significantly stronger in ACC than in the normal looking tissues $(P=0.000)$.

In the cribriform pattern of ACC, ABCG2 staining was observed on both cytoplasm and membrane of the epithelial cells. The highest intensity of ABCG2 staining was mainly localized in the periphery of cancer nests (Figure 2(a)). A similar pattern of ABCG2-positive staining in stromal vascular endothelial cells was also observed in the normal looking salivary gland tissues. In tubular pattern, ABCG2 was expressed in glandular epithelial cells (Figure 3(a)). In solid pattern, the myoepithelial cells were diffusely positive for ABCG2, and the highest intensity of ABCG2 staining was found focally in both periphery and center of cancer nests (Figure 4(a)).

CD133. CD133-positive cells were detected in 25 cases (1 case with weak intensity, 12 cases with moderate intensity, 


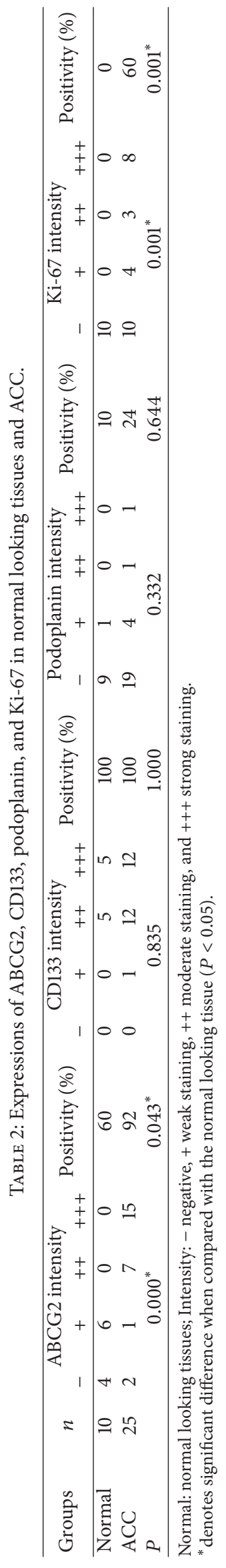




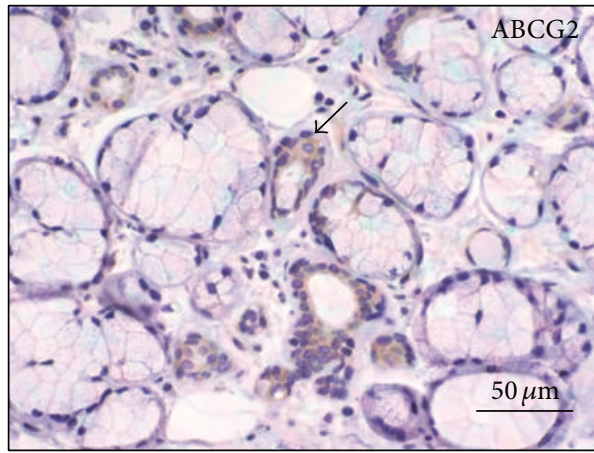

(a)

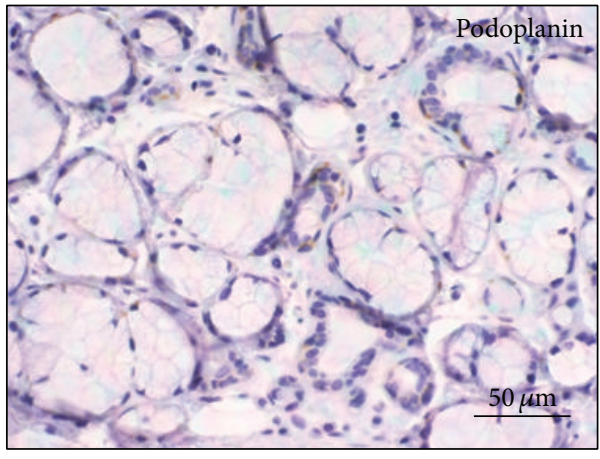

(c)

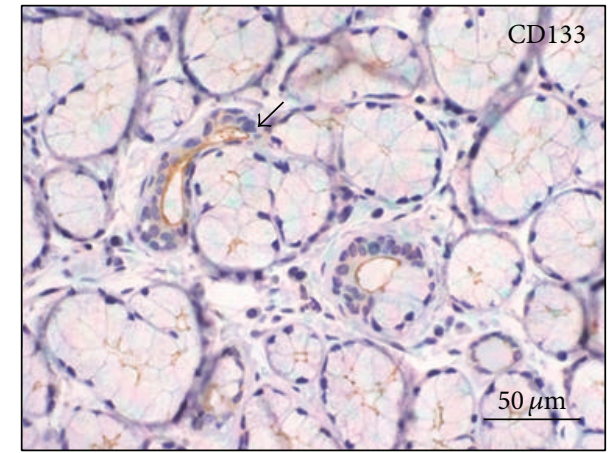

(b)

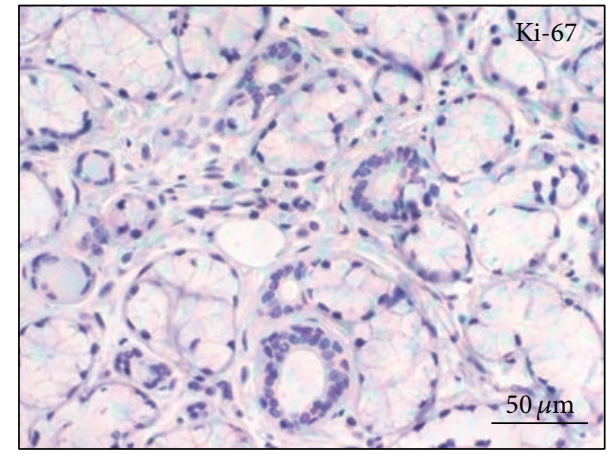

(d)

Figure 1: Expression of (a) ABCG2, (b) CD133, (c) podoplanin, and (d) Ki-67 in normal looking tissues.

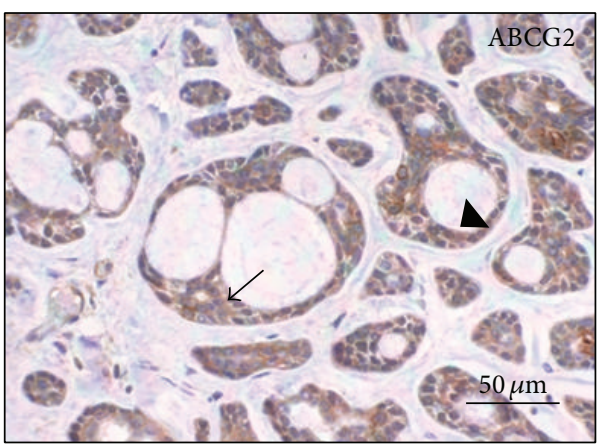

(a)

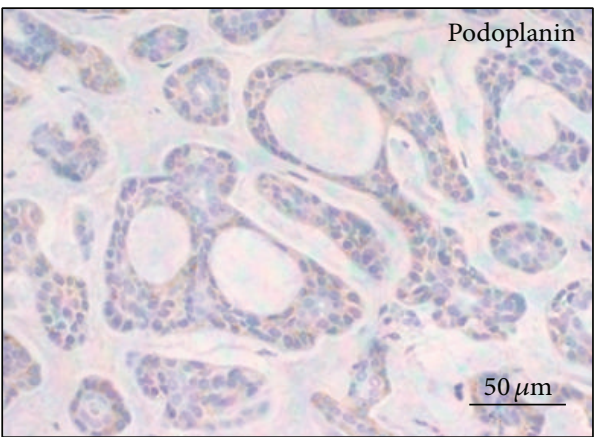

(c)

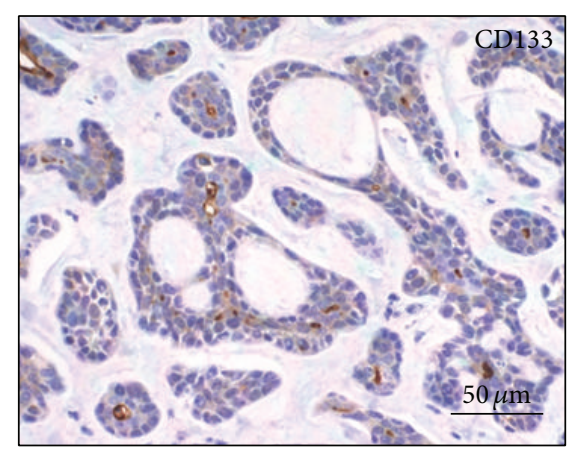

(b)

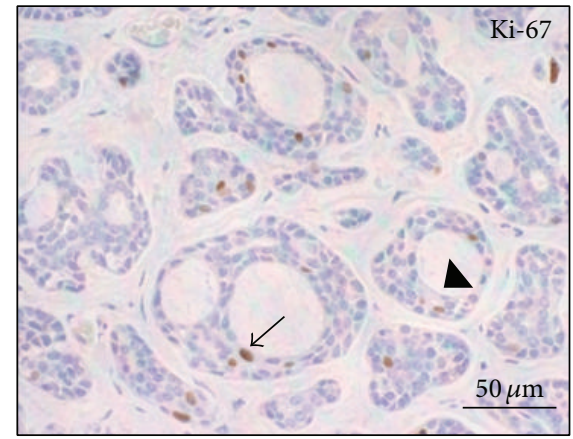

(d)

Figure 2: Expression of (a) ABCG2, (b) CD133, (c) podoplanin, and (d) Ki-67 in ACC of cribriform pattern. 


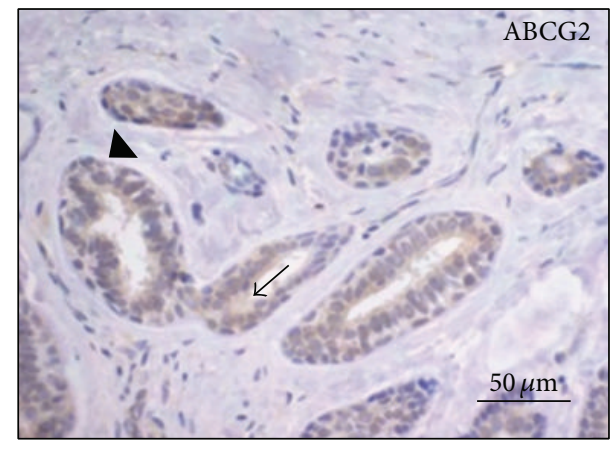

(a)

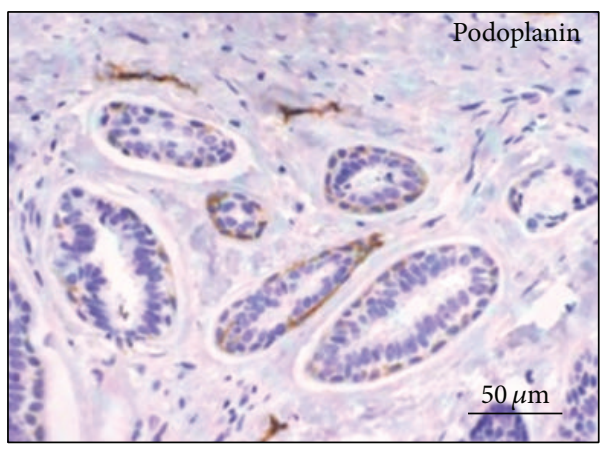

(c)

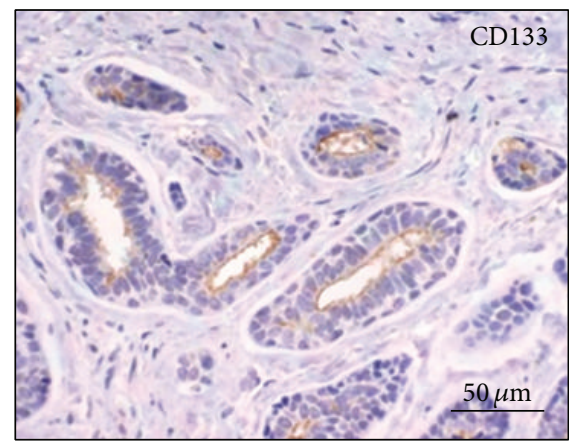

(b)

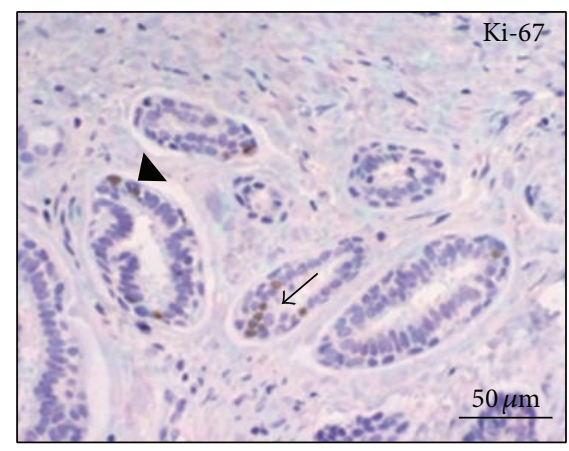

(d)

Figure 3: Expression of (a) ABCG2, (b) CD133, (c) podoplanin, and (d) Ki-67 in ACC of tubular pattern.

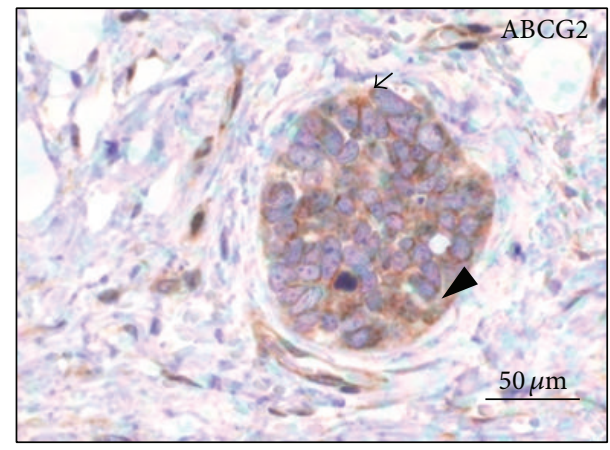

(a)

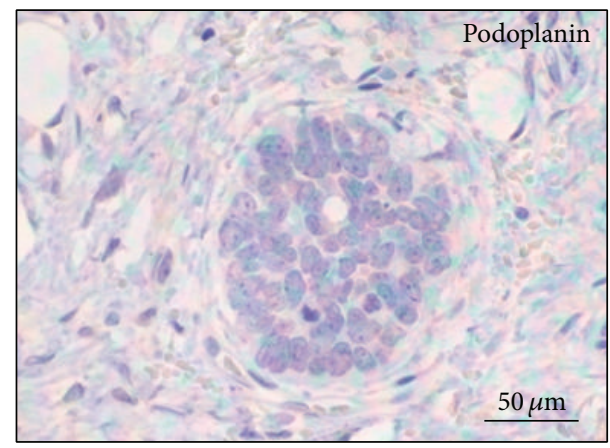

(c)

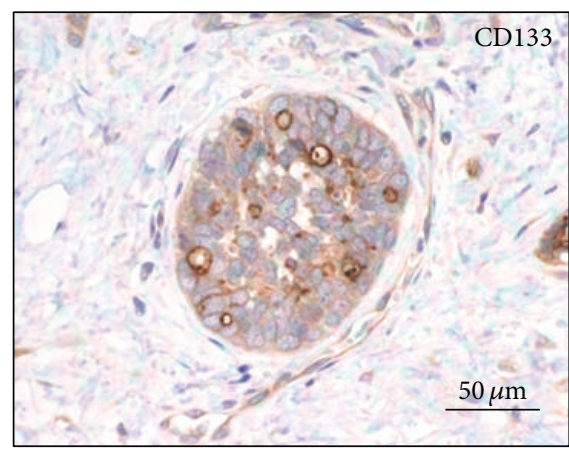

(b)

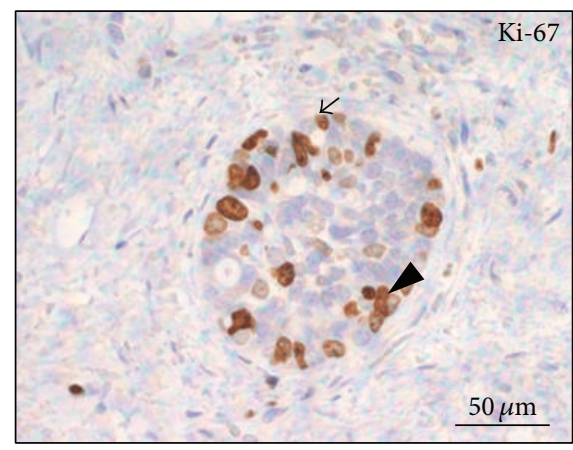

(d)

FIgUre 4: Expression of (a) ABCG2, (b) CD133, (c) podoplanin, and (d) Ki-67 in ACC of solid pattern. 


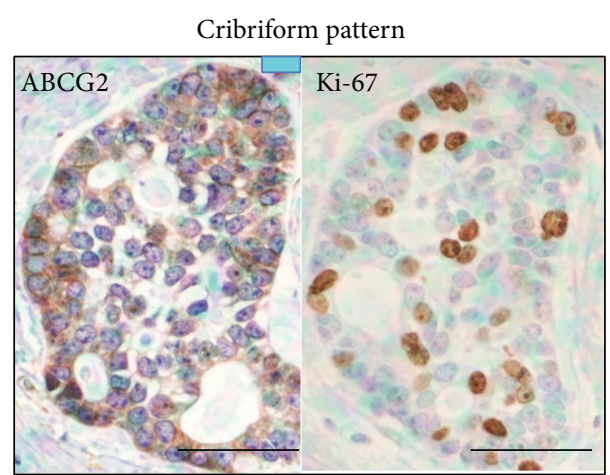

(a)

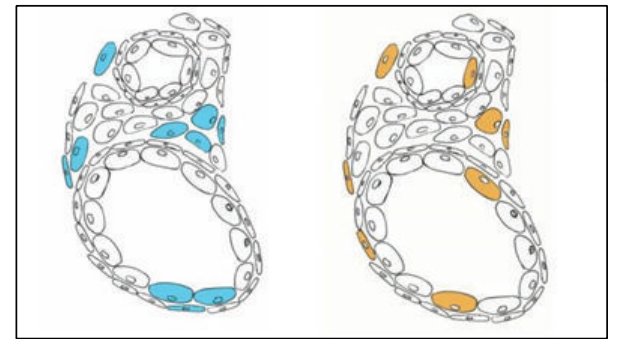

ABCG2

Ki-67

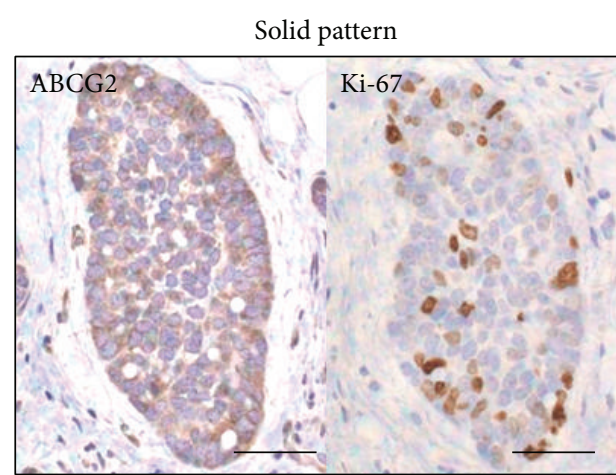

(b)

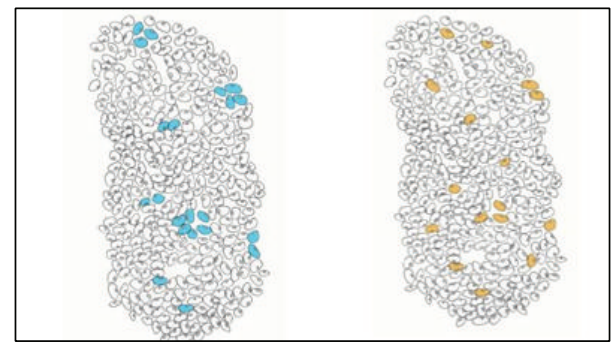

ABCG2

Ki-67

(c)

(d)

FIgURE 5: Positive expressions of ABCG2 and Ki-67 by the tumor cells were shown in the same area. The double positive stained cells were concentrated mainly in the peripheral zone of the cribriform pattern and also in the periphery and central zones of the solid pattern. Only a few strongly positive ABCG2 cells were negative for Ki-67 (scale bar: $50 \mu \mathrm{m}$ ).

and 12 cases with strong intensity) but limited in the apical membrane of the luminal cells in cribriform (Figure 2(b)) and tubular (Figure 3(b)) patterns. The contents in the ductal structures and pseudocyst spaces were positive for CD133. In solid pattern, positive expression was mainly diffused among the dense myoepithelial cells (Figure 4(b)). There was no significant difference of CD133 intensity or positivity between ACC and normal looking tissues $(P=0.835$ and $P=1.000$, resp.).

Podoplanin. Podoplanin expressions were detected in 6 cases of ACC including 4 cases with weak staining intensity, 1 case with moderate intensity, and 1 case with strong intensity, and the average positive percentage was $24 \%(P=0.644)$. The intensity in ACC showed no significant difference compared with the normal looking tissues $(P=0.332)$. Podoplanin can be only observed in myoepithelial cells and lymphatic endothelial cells. In most of the samples of cribriform pattern, podoplanin showed negative staining (Figure 2(c)). In tubular pattern of ACC, expressions of podoplanin were mainly detected in myoepithelial cells but not in glandular epithelial cells (Figure 3(c)). Negative podoplanin expressions were detected in all solid pattern samples (Figure 4(c)).

Ki-67. Positive expressions of Ki-67 were found in 15 ACC samples including 4 cases with weak staining intensity, 3 cases with moderate intensity, and 8 cases with strong
TABLE 3: The correlation information of ABCG2, CD133, and podoplanin in normal looking tissues.

\begin{tabular}{lcccc}
\hline & \multicolumn{2}{c}{ CD133 } & \multicolumn{2}{c}{ Podoplanin } \\
& $r_{s}$ & $P$ & $r_{s}$ & $P$ \\
\hline ABCG2 & 0.298 & 0.403 & 0.124 & 0.732 \\
CD133 & - & - & -0.333 & 0.347 \\
\hline
\end{tabular}

intensity. The average positive percentage was about $60 \%$ $(P=0.001)$, which was significantly stronger compared to the normal looking tissues $(P=0.001)$. The Ki-67positive stainings separated broadly in the tumor tissues of cribriform (Figure 2(d)) and solid pattern (Figure 4(d)). We also noticed that most cells overlaid with ABCG2 and Ki-67 were mainly found in the same location of the tumor tissue. A few cells expressing high level of ABCG2 with Ki-67negative expression were surrounded by cells expressing both ABCG2 and Ki-67 (Figure 5).

3.3. Statistical Results. The distribution correlation of ABCG2 and Ki-67 in ACC was summarized in Figure 5. We also compared the expression correlations of different markers between ACC and normal looking tissues (Table 2). To further identify the expression correlations among ABCG2, CD133, podoplanin, and Ki-67 in normal looking 
TABLE 4: The correlation information of ABCG2, CD133, podoplanin, and Ki-67 in ACC.

\begin{tabular}{lccccccc}
\hline & & CD133 & & \multicolumn{2}{c}{ Podoplanin } & \multicolumn{2}{c}{ Ki-67 } \\
& $r_{s}$ & & $P$ & $r_{s}$ & $P$ & $r_{s}$ & 0.620 \\
ABCG2 & -0.320 & - & 0.119 & 0.155 & 0.459 & $0.001^{*}$ \\
CD133 & - & - & 0.237 & 0.254 & -0.340 & 0.096 \\
Podoplanin & - & - & - & - & 0.069 & 0.743 \\
\hline
\end{tabular}

* denotes significant difference $(P<0.05)$.

tissues and ACC, we summarized the expression patterns of each marker in Tables 3 and 4. In normal looking tissues, there were no significant correlations between the two markers $(P>0.05)$ (Table 3). In ACC, only ABCG2 and Ki-67 showed a significant correlation $\left(r_{s}=0.620\right.$ and $\left.P=0.001\right)$ and no other significant associations were found between any two of the four markers $(P>0.05)$ (Table 4$)$.

\section{Discussion}

To date, growing evidence has identified that CSC is the driving force of tumor initiation and progression in several tumor types $[36,37]$. If CSCs can be identified and isolated from tumors using specific markers, new therapies may be established by effectively targeting the CSCs and therefore may improve the clinical outcomes of cancer treatment.

In this study, we characterized the expression patterns of ABCG2, CD133, and podoplanin in ACC of minor salivary glands by immunohistochemistry staining. We found a significantly higher expression of ABCG2 in ACC than in normal looking salivary gland tissues. We also exploited $\mathrm{Ki}-67$ to detect the proliferative activities of tumor cells in ACC, and we noticed that ABCG2 was also expressed in the same location with Ki-67-positive cells. These overlaid cells were mainly located in the peripheral zone of the cancer nest in cribriform pattern and in the periphery and central regions of the cancer nest in solid subtype. More interestingly, we noticed that a few cells with strong staining of ABCG2 but negative staining with $\mathrm{Ki}-67$ were usually surrounded by the cells expressing both ABCG2 and Ki-67. ABCG2 is widely expressed in many normal vital organs such as liver, lung, spleen, kidney, brain, nerve, skeletal muscle, cardiac muscle, testis, pancreas, small intestine, and trachea and also in a variety of tumors without tissue specificity [38, 39]. Meanwhile, ABCG2 shows dominant membrane staining in a wide range of stem cells [40] and is recognized as a universal marker for stem cells [41]. In this study, we found a diffusion expression pattern of ABCG2 in ACC and the expression level in ACC is significantly higher than that in normal looking tissues which suggested that ABCG 2 might be involved in the development process of ACC and played a role in enhancing the proliferation and invasion capabilities of cancer cells. Compared to the expression pattern of Ki67, we also noticed that most ABCG2-positive cells also had positive staining with Ki-67 and mainly located in the periphery of cribriform cancer nests and in both periphery and central zones of solid cancer nests.

It is known that Ki-67, as a marker of cell proliferation, is absent in quiescent cells but has universal expression in proliferating cells [42]. According to the CSC theory, CSCs are rare and quiescent and have the potential of selfrenewing and maintaining tumor growth and heterogeneity [43]. Some studies also reported that the Ki-67-positive cells are less likely to contain cancer stem-like cells due to their high degree of proliferation and differentiation activity [41]. Conversely, ABCG2 is found to be overexpressed in SP stem cells isolated form bone marrow [44] and a number of established cancer cell lines as well as tumor samples [4547]. ABCG2 is also speculated to be critical in maintaining a quiescent state of the stem cells although the mechanism is still unclear [48]. From our results, a small number of cells with the ABCG2 strongest staining and Ki-67-negative staining (ABCG2+++/Ki-67-) could be repetitively found to be surrounded with cells expressing both ABCG2 and $\mathrm{Ki}-67$. We speculate that cancer stem-like cells may exist in the cells with ABCG2+++/Ki-67- staining, which are surrounded by cells expressing both ABCG2 and Ki-67 in ACC and ABCG2 may play a role in the process of cellular proliferation and development in ACC. Our findings suggest that costaining with ABCG2 and Ki-67 may help predict the location of CSCs in ACC. Besides, the statistical analysis based on the total cases of ACC analyzed in this study showed a significant correlation between ABCG2 and Ki67 , which can further support our above findings. However, our sample size is not big enough for the statistical analysis according to the sample varies based on the clinical and histopathological classifications. More samples are needed in the future studies to identify the above findings based on sample clinical and histopathological varies and additionally determine the mechanism of their correlations.

We also explored the expression patterns of ABCG2, CD133, and podoplanin in normal looking and ACC tissues. We found that ABCG2 and CD133 had a very similar positivity in ACC (92\% and $100 \%$, resp.); however, the positive staining distributions of ABCG2 and CD133 in ACC were different. In the cribriform pattern of ACC, ABCG2 stainings were observed in cytoplasm and membrane of epithelial cells. In the tubular pattern, ABCG2 was expressed in glandular epithelial cells. In the solid pattern, the myoepithelial cells were diffusely positive for ABCG2. CD133 staining could only be found in the luminal cells of cribriform and tubular patterns. In the solid pattern, CD133-positive expression was mainly diffused among the dense myoepithelial cells. There was no significant correlation between CD133 and ABCG2 or $\mathrm{Ki}-67$ based on their staining intensity and location. CD133 has been studied in recent years as a specific surface molecule in detecting CSCs and CD133-positive cancer cells which are also found possessing many stem cell characteristics 
$[7,25,49]$. Its attentiveness as a cancer stem cell marker has recently grown dramatically in breast cancer $[17,50]$, brain cancer [21], prostate cancer [22], pancreatic cancer [22], kidney cancer [51], and also in hepatocarcinoma [25] and melanoma [52]. In our study, we also noticed a similar expression pattern of CD133 in the normal looking tissues of human minor salivary glands as reported in the mammary glands, pancreas, and liver [53]. However, the CD133 expression pattern and intensity were similar in both normal looking tissues and in ACC from our study so that no definitive CD133-positive tumor cells could be distinguished in our ACC samples.

Podoplanin has also been investigated for its potential as a CSC marker in the solid tumors in recent studies. Podoplanin is found in various neoplasms, such as squamous-cell carcinoma [54], germ cell tumors [55], mesothelioma [56], and some subtypes of vascular tumors. Podoplanin is also considered to be involved in the process of tumor cell migration, invasion, and metastasis. From our results, podoplanin only exhibited weak positive staining in the cytoplasm and cell membrane of myoepithelial cells of normal looking tissues and cribriform and tubular patterns of ACC, but showed negative staining in solid pattern of ACC. Furthermore, we did not find any significant difference of podoplanin expressions between the normal looking tissues and ACC. All these results are not sufficient enough to support that CD133 or podoplanin can detect cancer stem cells in ACC. We speculate that a more accurate and specific way to quickly locate and identify the CSCs with clinical carcinoma samples is to target the candidate cells by costaining with multiple CSC markers. If CSCs can be successfully located, anticancer drugs can be precisely delivered into the cancer nest through tissue engineering technologies and the efficiency of the current clinical treatments can be significantly improved. Therefore, finding out the expression patterns and correlations of the CSC surface markers with immunohistological studies is an important and initial step towards this goal. In our study, we did not find out any significant associations among the investigated markers (ABCG2, CD133, and podoplanin) in ACC. More efforts will be put in exploring the expression patterns and correlations of other commonly used CSC markers such as CD44, CD24, CD29, CD90, and aldehyde dehydrogenase 1 (ALDH1) in ACC of minor salivary glands. Still, whether CD133 or podoplanin can be selected as a specific marker of CSCs in ACC and its biological and functional contribution to tumorigenesis needs to be answered in further investigations.

\section{Conclusion}

In conclusion, the expression of ABCG2 showed more significant upregulation in ACC than in normal looking tissues and it showed a similar expression pattern with $\mathrm{Ki}-67$ in ACC. We speculate that the cancer stem-like cells may exist with ABCG2+++/Ki-67- staining, which are surrounded by cells with positive expression of ABCG2 and Ki-67 in ACC. Costaining with ABCG2 and Ki-67 may help predict the location of CSCs in ACC.
In future studies, we will study more clinical samples based on clinical and histopathological classification to find out the relationships between the expression level of ABCG2 and other CSC markers and the prognosis and clinical implications of ACC patients. We will explore the expression patterns and correlations of other surface markers that are commonly used in identifying CSCs such as CD44, CD24, CD29, CD90, and aldehyde dehydrogenase 1 (ALDH1) in ACC of minor salivary glands.

\section{Conflict of Interests}

The authors declare that there is no conflict of interests regarding the publication of this paper.

\section{Acknowledgments}

This work was supported by JSPS KAKENHI Grant nos. 24592766 and 25463084 . The authors thank Japanese International Cooperation Agency (JICA) for the financial support and also all the faculty members in Department of Oral Pathology and Medicine, Graduate School of Medicine, Dentistry and Pharmaceutical Sciences of Okayama University for their support in experimental samples and facilities.

\section{References}

[1] P. J. Bradley, "Adenoid cystic carcinoma of the head and neck: a review," Current Opinion in Otolaryngology and Head and Neck Surgery, vol. 12, no. 2, pp. 127-132, 2004.

[2] P. J. Giannini, K. V. Shetty, S. L. Horan, W. D. Reid, and L. L. Litchmore, "Adenoid cystic carcinoma of the buccal vestibule: a case report and review of the literature," Oral Oncology, vol. 42, no. 10, pp. 1029-1032, 2006.

[3] J. D. Casler and J. J. Conley, "Surgical management of adenoid cystic carcinoma in the parotid gland," Otolaryngology-Head and Neck Surgery, vol. 106, no. 4, pp. 332-338, 1992.

[4] I. L. Weissman, "Stem cells: units of development, units of regeneration, and units in evolution," Cell, vol. 100, no. 1, pp. 157-168, 2000.

[5] J. Lessard and G. Sauvageau, "Bmi-1 determines the proliferative capacity of normal and leukaemic stem cells," Nature, vol. 423, no. 6937, pp. 255-260, 2003.

[6] M. Al-Hajj, M. S. Wicha, A. Benito-Hernandez, S. J. Morrison, and M. F. Clarke, "Prospective identification of tumorigenic breast cancer cells," Proceedings of the National Academy of Sciences of the United States of America, vol. 100, no. 7, pp. 39833988, 2003.

[7] S. K. Singh, I. D. Clarke, M. Terasaki et al., "Identification of a cancer stem cell in human brain tumors," Cancer Research, vol. 63, no. 18, pp. 5821-5828, 2003.

[8] S. Takaishi, T. Okumura, S. Tu et al., "Identification of gastric cancer stem cells using the cell surface marker CD44," Stem Cells, vol. 27, no. 5, pp. 1006-1020, 2009.

[9] J. Burkert, N. A. Wright, and M. R. Alison, "Stem cells and cancer: an intimate relationship," Journal of Pathology, vol. 209, no. 3, pp. 287-297, 2006.

[10] M. Kim, H. Turnquist, J. Jackson et al., “The multidrug resistance transporter ABCG2 (breast cancer resistance protein 1) 
effluxes Hoechst 33342 and is overexpressed in hematopoietic stem cells," Clinical Cancer Research, vol. 8, no. 1, pp. 22-28, 2002.

[11] I. L. Botchkina, R. A. Rowehl, D. E. Rivadeneira et al., "Phenotypic subpopulations of metastatic colon cancer stem cells: genomic analysis," Cancer Genomics and Proteomics, vol. 6, no. 1, pp. 19-30, 2009.

[12] E. C. Anderson, C. Hessman, T. G. Levin, M. M. Monroe, and M. $\mathrm{H}$. Wong, "The role of colorectal cancer stem cells in metastatic disease and therapeutic response," Cancers, vol. 3, no. 1, pp. 319339, 2011.

[13] L. Du, H. Wang, and L. He, "CD44 is of functional importance for colorectal cancer stem cells," Clinical Cancer Research, vol. 14, no. 21, pp. 6751-6760, 2008.

[14] E. L.-H. Leung, R. R. Fiscus, J. W. Tung et al., "Non-small cell lung cancer cells expressing CD44 are enriched for stem celllike properties," PLoS ONE, vol. 5, no. 11, Article ID e14062, 2010.

[15] H. J. Lee, G. Choe, S. Jheon, S.-W. Sung, C.-T. Lee, and J.-H. Chung, "CD24, a novel cancer biomarker, predicting diseasefree survival of non-small cell lung carcinomas: a retrospective study of prognostic factor analysis from the viewpoint of forthcoming (Seventh) New TNM classification," Journal of Thoracic Oncology, vol. 5, no. 5, pp. 649-657, 2010.

[16] P. A. Fetsch, A. Abati, T. Litman et al., "Localization of the ABCG2 mitoxantrone resistance-associated protein in normal tissues," Cancer Letters, vol. 235, no. 1, pp. 84-92, 2006.

[17] S. Zhou, J. D. Schuetz, K. D. Bunting et al., "The ABC transporter Bcrp1/ABCG2 is expressed in a wide variety of stem cells and is a molecular determinant of the side-population phenotype," Nature Medicine, vol. 7, no. 9, pp. 1028-1034, 2001.

[18] A.-M. Bleau, D. Hambardzumyan, T. Ozawa et al., "PTEN/ PI3K/Akt pathway regulates the side population phenotype and ABCG2 activity in glioma tumor stem-like cells," Cell Stem Cell, vol. 4, no. 3, pp. 226-235, 2009.

[19] X.-W. Ding, J.-H. Wu, and C.-P. Jiang, "ABCG2: a potential marker of stem cells and novel target in stem cell and cancer therapy," Life Sciences, vol. 86, no. 17-18, pp. 631-637, 2010.

[20] S. Miraglia, W. Godfrey, A. H. Yin et al., "A novel fivetransmembrane hematopoietic stem cell antigen: isolation, characterization, and molecular cloning," Blood, vol. 90, no. 12, pp. 5013-5021, 1997.

[21] T. Chiba, K. Kita, Y.-W. Zheng et al., "Side population purified from hepatocellular carcinoma cells harbors cancer stem celllike properties," Hepatology, vol. 44, no. 1, pp. 240-251, 2006.

[22] M. D. Brown, P. E. Gilmore, C. A. Hart et al., "Characterization of benign and malignant prostate epithelial Hoechst 33342 side populations," Prostate, vol. 67, no. 13, pp. 1384-1396, 2007.

[23] S. K. Singh, C. Hawkins, I. D. Clarke et al., "Identification of human brain tumour initiating cells," Nature, vol. 432, no. 7015, pp. 396-401, 2004.

[24] A. T. Collins, P. A. Berry, C. Hyde, M. J. Stower, and N. J. Maitland, "Prospective identification of tumorigenic prostate cancer stem cells," Cancer Research, vol. 65, no. 23, pp. 1094610951, 2005.

[25] S. Yin, J. Li, C. Hu et al., "CD133 positive hepatocellular carcinoma cells possess high capacity for tumorigenicity," International Journal of Cancer, vol. 120, no. 7, pp. 1444-1450, 2007.

[26] A. Eramo, F. Lotti, G. Sette et al., "Identification and expansion of the tumorigenic lung cancer stem cell population," Cell Death and Differentiation, vol. 15, no. 3, pp. 504-514, 2008.
[27] M. Todaro, M. P. Alea, A. B. Di Stefano et al., "Colon cancer stem cells dictate tumor growth and resist cell death by production of interleukin-4," Cell Stem Cell, vol. 1, no. 4, pp. 389-402, 2007.

[28] S.-H. Chiou, C.-C. Yu, C.-Y. Huang et al., "Positive correlations of Oct-4 and Nanog in oral cancer stem-like cells and highgrade oral squamous cell carcinoma," Clinical Cancer Research, vol. 14, no. 13, pp. 4085-4095, 2008.

[29] Q. Zhang, S. Shi, Y. Yen, J. Brown, J. Q. Ta, and A. D. Le, “A subpopulation of CD133+ cancer stem-like cells characterized in human oral squamous cell carcinoma confer resistance to chemotherapy," Cancer Letters, vol. 289, no. 2, pp. 151-160, 2010.

[30] M. Kreppel, M. Scheer, U. Drebber, L. Ritter, and J. E. Zöller, "Impact of podoplanin expression in oral squamous cell carcinoma: clinical and histopathologic correlations," Virchows Archiv, vol. 456, no. 5, pp. 473-482, 2010.

[31] E. Martín-Villar, F. G. Scholl, C. Gamallo et al., "Characterization of human PA2.26 antigen (T1 $\alpha$-2, podoplanin), a small membrane mucin induced in oral squamous cell carcinomas," International Journal of Cancer, vol. 113, no. 6, pp. 899-910, 2005.

[32] E. Martín-Villar, D. Megías, S. Castel, M. M. Yurrita, S. Vilaró, and M. Quintanilla, "Podoplanin binds ERM proteins to activate RhoA and promote epithelial-mesenchymal transition," Journal of Cell Science, vol. 119, no. 21, pp. 4541-4553, 2006.

[33] K. Mishima, Y. Kato, M. K. Kaneko, R. Nishikawa, T. Hirose, and M. Matsutani, "Increased expression of podoplanin in malignant astrocytic tumors as a novel molecular marker of malignant progression," Acta Neuropathologica, vol. 111, no. 5, pp. 483-488, 2006.

[34] N. Atsumi, G. Ishii, M. Kojima, M. Sanada, S. Fujii, and A. Ochiai, "Podoplanin, a novel marker of tumor-initiating cells in human squamous cell carcinoma A431," Biochemical and Biophysical Research Communications, vol. 373, no. 1, pp. 36-41, 2008.

[35] N. Rahadiani, J.-I. Ikeda, T. Makino et al., "Tumorigenic role of podoplanin in esophageal squamous-cell carcinoma," Annals of Surgical Oncology, vol. 17, no. 5, pp. 1311-1323, 2010.

[36] T. Reya, S. J. Morrison, M. F. Clarke, and I. L. Weissman, "Stem cells, cancer, and cancer stem cells," Nature, vol. 414, no. 6859, pp. 105-111, 2001.

[37] H. Kitamura, K. Okudela, T. Yazawa, H. Sato, and H. Shimoyamada, "Cancer stem cell: Implications in cancer biology and therapy with special reference to lung cancer," Lung Cancer, vol. 66 , no. 3, pp. 275-281, 2009.

[38] M. Maliepaard, G. L. Scheffer, I. F. Faneyte et al., "Subcellular localization and distribution of the Breast Resistance Protein Transporter in normal human tissues," Cancer Research, vol. 61, no. 8, pp. 3458-3464, 2001.

[39] M. Huls, C. D. A. Brown, A. S. Windass et al., "The breast cancer resistance protein transporter ABCG2 is expressed in the human kidney proximal tubule apical membrane," Kidney International, vol. 73, no. 2, pp. 220-225, 2008.

[40] J. E. Trosko and R. J. Ruch, "Cell-cell communication in carcinogenesis," Frontiers in Bioscience, vol. 3, pp. D208-D236, 1998.

[41] G. A. Sathi, R. Tamamura, H. Tsujigiwa et al., "Analysis of immunoexpression of common cancer stem cell markers in ameloblastoma," Experimental and Therapeutic Medicine, vol. 3, no. 3, pp. 397-402, 2012.

[42] J. H. Van Dierendonck, R. Keijzer, C. J. H. Van de Velde, and C. J. Cornelisse, "Nuclear distribution of the Ki-67 antigen during the cell cycle: comparison with growth fraction in human breast 
cancer cells," Cancer Research, vol. 49, no. 11, pp. 2999-3006, 1989.

[43] V. Tirino, V. Desiderio, F. Paino et al., "Cancer stem cells in solid tumors: an overview and new approaches for their isolation and characterization," The FASEB Journal, vol. 27, no. 1, pp. 13-24, 2013.

[44] S. Zhou, J. J. Morris, Y. Barnes, L. Lan, J. D. Schuetz, and B. P. Sorrentino, "Bcrpl gene expression is required for normal numbers of side population stem cells in mice, and confers relative protection to mitoxantrone in hematopoietic cells in vivo," Proceedings of the National Academy of Sciences of the United States of America, vol. 99, no. 19, pp. 12339-12344, 2002.

[45] C. Hirschmann-Jax, A. E. Foster, G. G. Wulf et al., "A distinct "side population" of cells with high drug efflux capacity in human tumor cells," Proceedings of the National Academy of Sciences of the United States of America, vol. 101, no. 39, pp. 14228-14233, 2004.

[46] N. Haraguchi, H. Ishii, K. Mimori et al., "CD13 is a therapeutic target in human liver cancer stem cells," Journal of Clinical Investigation, vol. 120, no. 9, pp. 3326-3339, 2010.

[47] M. Olempska, P. A. Eisenach, O. Ammerpohl, H. Ungefroren, F. Fandrich, and H. Kalthoff, "Detection of tumor stem cell markers in pancreatic carcinoma cell lines," Hepatobiliary and Pancreatic Diseases International, vol. 6, no. 1, pp. 92-97, 2007.

[48] O. Tsinkalovsky, A. O. Vik-Mo, S. Ferreira, O. D. Laerum, and A. Fjose, "Zebrafish kidney marrow contains ABCG2dependent side population cells exhibiting hematopoietic stem cell properties," Differentiation, vol. 75, no. 3, pp. 175-183, 2007.

[49] H. You, W. Ding, and C. B. Rountree, "Epigenetic regulation of cancer stem cell marker CD133 by transforming growth factor$\beta$,' Hepatology, vol. 51, no. 5, pp. 1635-1644, 2010.

[50] T. Kondo, T. Setoguchi, and T. Taga, "Persistence of a small subpopulation of cancer stem-like cells in the C6 glioma cell line," Proceedings of the National Academy of Sciences of the United States of America, vol. 101, no. 3, pp. 781-786, 2004.

[51] N. H. Bander, C. L. Finstad, C. Cordon-Cardo et al., "Analysis of a mouse monoclonal antibody that reacts with a specific region of the human proximal tubule and subsets renal cell carcinomas," Cancer Research, vol. 49, no. 23, pp. 6774-6780, 1989.

[52] E. Monzani, F. Facchetti, E. Galmozzi et al., "Melanoma contains CD133 and ABCG2 positive cells with enhanced tumourigenic potential," European Journal of Cancer, vol. 43, no. 5, pp. 935946, 2007.

[53] T. Welsch, S. Keleg, F. Bergmann, L. Degrate, S. Bauer, and J. Schmidt, "Comparative analysis of tumorbiology and CD133 positivity in primary and recurrent pancreatic ductal adenocarcinoma," Clinical and Experimental Metastasis, vol. 26, no. 7, pp. 701-711, 2009.

[54] Y. Kato, M. Kaneko, M. Sata, N. Fujita, T. Tsuruo, and M. Osawa, "Enhanced expression of aggrus (Tlalpha/podoplanin), a platelet-aggregation-inducing factor in lung squamous cell carcinoma," Tumor Biology, vol. 26, no. 4, pp. 195-200, 2005.

[55] K. Mishima, Y. Kato, M. K. Kaneko et al., "Podoplanin expression in primary central nervous system germ cell tumors: a useful histological marker for the diagnosis of germinoma," Acta Neuropathologica, vol. 111, no. 6, pp. 563-568, 2006.

[56] N. Kimura and I. Kimura, "Podoplanin as a marker for mesothelioma," Pathology International, vol. 55, no. 2, pp. 83-86, 2005. 


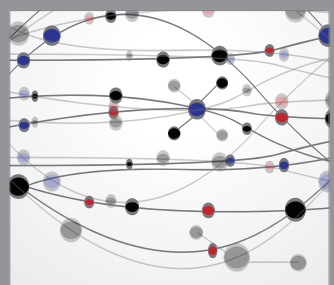

The Scientific World Journal
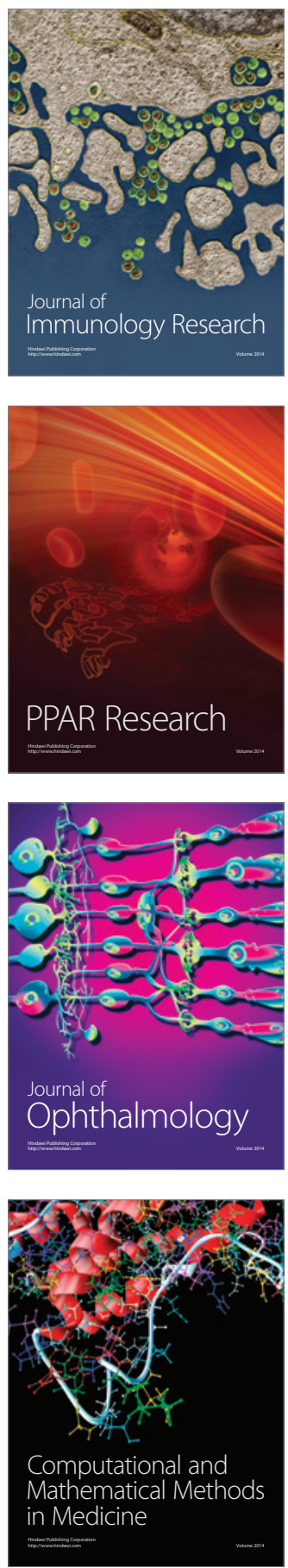

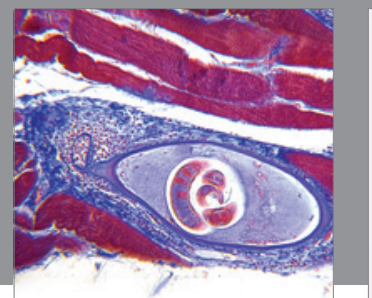

Gastroenterology

Research and Practice
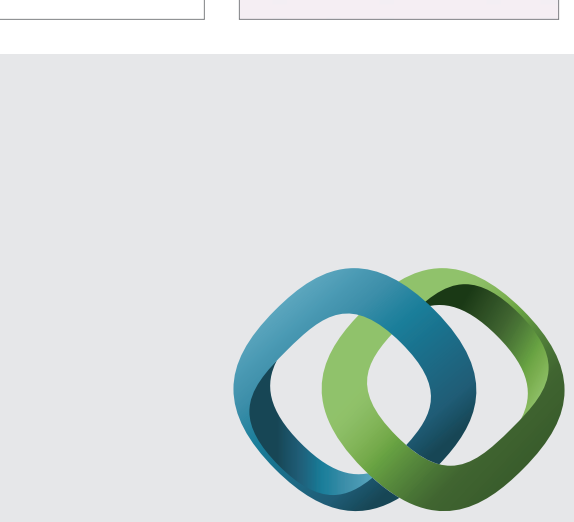

\section{Hindawi}

Submit your manuscripts at

http://www.hindawi.com
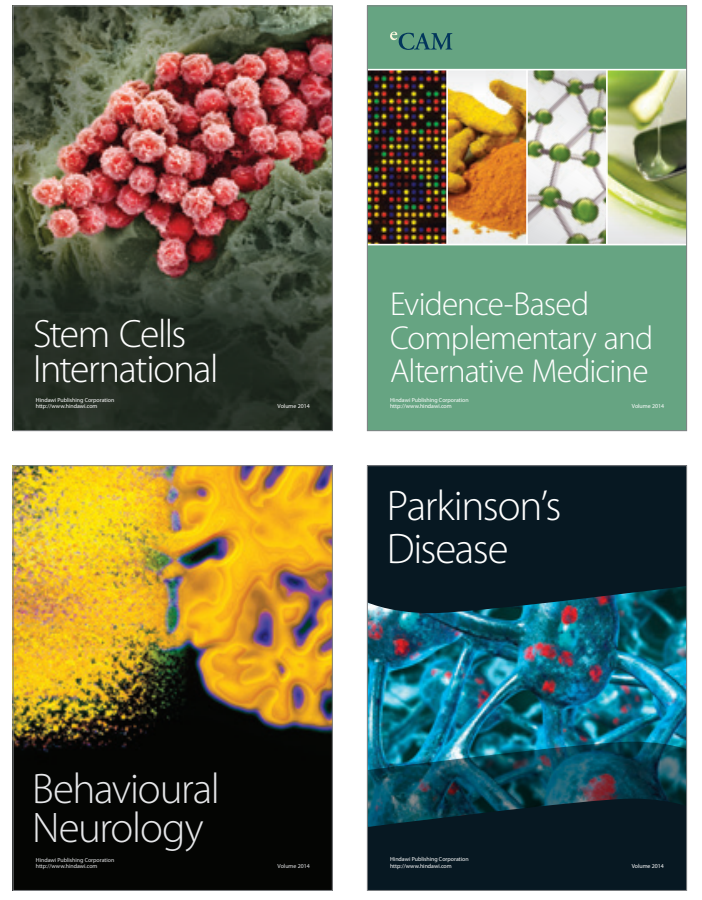
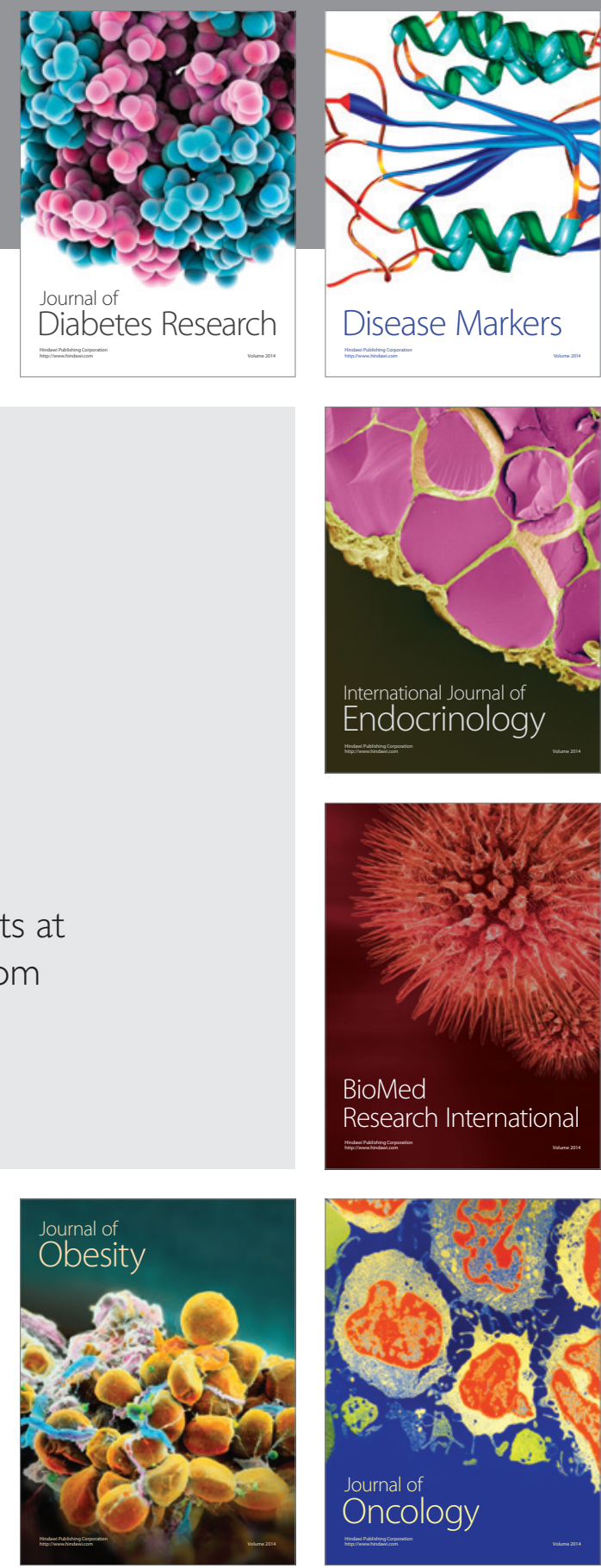

Disease Markers
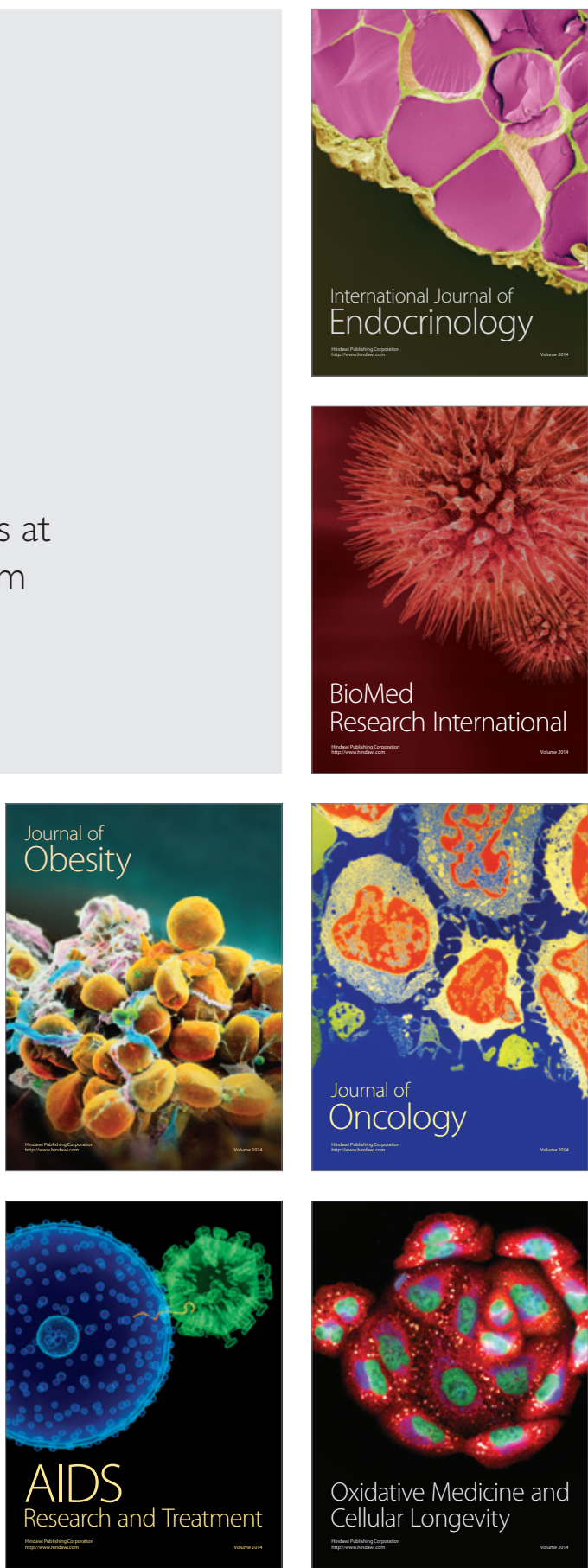